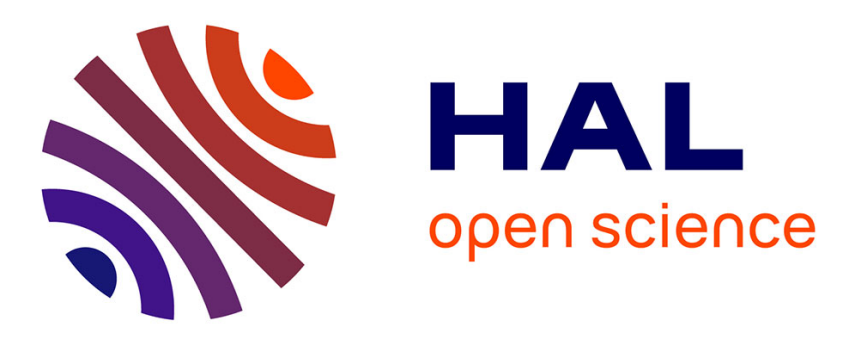

\title{
Shaping the spontaneous emission of extended incoherent sources into composite radial vector beams
}

Domitille Schanne, Stéphan Suffit, Pascal Filloux, Emmanuel Lhuillier, Aloyse

Degiron

\section{- To cite this version:}

Domitille Schanne, Stéphan Suffit, Pascal Filloux, Emmanuel Lhuillier, Aloyse Degiron. Shaping the spontaneous emission of extended incoherent sources into composite radial vector beams. Applied Physics Letters, 2021, 119, 10.1063/5.0065486 . hal-03412641

\section{HAL Id: hal-03412641 \\ https://hal.science/hal-03412641}

Submitted on 3 Nov 2021

HAL is a multi-disciplinary open access archive for the deposit and dissemination of scientific research documents, whether they are published or not. The documents may come from teaching and research institutions in France or abroad, or from public or private research centers.
L'archive ouverte pluridisciplinaire HAL, est destinée au dépôt et à la diffusion de documents scientifiques de niveau recherche, publiés ou non, émanant des établissements d'enseignement et de recherche français ou étrangers, des laboratoires publics ou privés. 


\title{
Shaping the Spontaneous Emission of Extended Incoherent Sources into Composite Radial Vector Beams
}

\author{
Domitille Schanne ${ }^{1}$, Stéphan Suffit ${ }^{1}$, Pascal Filloux ${ }^{1}$, Emmanuel Lhuillier $^{2}$, Aloyse Degiron $^{1, a)}$ \\ ${ }^{1}$ Université de Paris, CNRS, Laboratoire Matériaux et Phénomènes Quantiques, F-75205 Paris, \\ France \\ ${ }^{2}$ Sorbonne Université, CNRS, Institut des NanoSciences de Paris, INSP, F-75005 Paris, France \\ a) Author to whom correspondence should be addressed: aloyse.degiron@u-paris.fr
}

\begin{abstract}
It is well known that concentric diffraction gratings are capable of beaming the spontaneous emission of large extended incoherent light sources (e.g. hot radiating surfaces and luminescent materials). Here, we reveal additional properties of such beams using layers of colloidal PbS nanocrystals coated onto metallic spiraling gratings as an example. We observe and explain with a simple model the formation of multiple beams when the spirals are deformed. We also point out an aspect of the light emission that does not seem to have been discussed so farnamely, that the polarization of the directional beams has a radial distribution. These findings are not restricted to our experimental configuration, suggesting a simple way to build incandescent and electroluminescent sources with non-trivial polarization states. The price to pay is an isotropic emission background due to the composite nature of the beams, which result from the incoherent superposition of a continuum of diffracted plasmons everywhere above the surface.
\end{abstract}

$* * * * * * * * * * *$ 
Achieving a full control of the spontaneous emission of extended incoherent light sources, such as hot radiating surfaces or luminescent materials, poses challenges that are perhaps even more formidable than those encountered for shaping the luminescence of single emitters. The reason for these difficulties is that each point of an incoherent source emits electromagnetic radiation with a different phase that randomly varies over time-a behavior seemingly irreconcilable with the precise phase conditions required for the formation of directional and/or polarized beams. Research in this field is very active nowadays, with recent breakthroughs on unidirectional beaming and/or lensing of thermal ${ }^{1}$ and luminescent ${ }^{2,3}$ metasurfaces, the demonstration of highly directional and narrowband absorption (which is equivalent to emission) in photonic crystals featuring a perturbed Dirac-cone band structure ${ }^{4}$, the use of disordered and aperiodic structures to shape the fluorescence of dye layers ${ }^{5,6}$, as well as theoretical proposals for the full control of thermal emission with metasurfaces leveraging both local and non-local interactions ${ }^{7}$.

Moreover, it was recognized decades ago that integrating a linear diffraction grating into an incoherent source leads to anisotropic, and in some cases, polarized emission ${ }^{8-10}$. Despite the wide variety of cases considered over the years, the basic ingredients for shaping the emission with a linear grating have not changed. First, one must couple the photons to a guided (and at least partially spatially coherent) electromagnetic mode that propagates along the plane of the light-emitting surface with a well-defined wavevector $\mathbf{k}$ (e.g. a surface wave ${ }^{8-10}$, or an optical mode within a dielectric layer $\left.{ }^{11}\right)$. Second, one perturbs this mode with a periodic grating imprinted on the structure, inducing a directional leakage of the electromagnetic energy into free space. The origin of the directionality is that photons that are diffracted by the grating have an in-plane wavevector component $\mathbf{k}_{/ /}$that must satisfy the laws of energy and momentum conservation: $\mathbf{k}_{/ /}=\mathbf{k} \pm \mathbf{G}$, where $\mathbf{G}$ is a reciprocal vector, which is itself proportional to the inverse of the pitch of the grating. The 
value of $\left|k_{/}\right| \mid=k_{0} \sin \theta$ determines the angle $\theta$ at which the photons with wavelength $\lambda=2 \pi / k_{0}$ are emitted with respect to the normal to the surface. The resulting lobes always come in pairs, except if the unit cells of the grating are carefully engineered to make the emission unidirectional ${ }^{1}$. When the guided mode involved in the process is a TE- or TM-polarized surface wave, the resulting emission is linearly polarized along $\mathbf{k} / /^{12-14}$.

Despite these successes, the possibilities offered by linear gratings remain limited, if only because the resulting beams are not laterally confined in all directions of space. Furthermore, they cannot lead to non-trivial polarization states such as beams with radial or azimuthal field distributions. A few studies have considered two-dimensional gratings to address some of these shortcomings. In particular, deformed gratings have been shown to induce local variations of the linear polarization above the surface of a thermal source, although the resulting emission was not directional ${ }^{15}$. Another interesting development concerns the full collimation of the light produced by incoherent sources using circular gratings, i.e. bull's eye structures, as demonstrated by Norris and coworkers for thermal radiation and photoluminescence $(\mathrm{PL})^{16-18}$. To understand the origin of the collimation, one can consider that each point of the source emits light that interacts with a grating with a different local orientation. The net emission pattern results from the incoherent superposition of diffraction lobes whose direction and shape vary from one point of the surface to another. Due to the axial symmetry of the bull's eye structure, the only contribution that all these lobes can have in common is in the direction normal to the surface. Thus, a well-shaped composite beam perpendicular to the surface is obtained as a result of the energy build-up in this particular direction ${ }^{16}$. When the pitch of the bull's eye is adjusted so that $\mathbf{k} / /$ vanishes in the above equation, a directional beam with very narrow divergence is observed. Due to the dispersive nature of the grating, this condition is only fulfilled for one wavelength. As $\lambda$ shifts away from this optimal 
condition, the emitted light remains however shaped under the form of a hollow cone with an aperture defined by the norm of $\mathbf{k} / /$.

In this Letter, we discuss additional properties of such composite (or, shall we say, "meta") beams and apply this knowledge to make extended incoherent sources emit multiple beams and beams with non-trivial polarization textures. We begin our study by extending some results of the literature to the three-branch spiral of Fig. 1(a) — a variant of the bull's eye geometry that we have already used in a previous article to demonstrate the spontaneous emission of vector vortex beams ${ }^{19}$. The radial period is $1235 \mathrm{~nm}$, the height of the corrugations is $25 \mathrm{~nm}$ and the total footprint of the structure is a disk of $100 \mu \mathrm{m}$. This sample as well as all subsequent structures are fabricated on a silicon substrate covered by a $200 \mathrm{~nm}$ thick layer of gold with a sequence of electron beam lithography, gold evaporation and lift-off. A scanning electron microscope (SEM) image of the structure is displayed in Fig. 1(a). The spiral is coated by a 15-nm thick continuous layer of PbS colloidal nanocrystals (NCs) which were synthetized following the procedure of Moreels et al. ${ }^{20,21}$ (supplementary material). The near-infrared PL spectrum of the sample is shown in Fig. 1(b). We note that contrarily to the case of single emitters ${ }^{22}$, the PL is not completely quenched even though the NCs are in contact with the lossy metallic surface. This difference in behavior is a manifestation of the ensemble properties of the PbS NCs, as explained elsewhere ${ }^{23,24}$.

Figure 2 illustrates how the angular distribution $\left|\mathbf{k}_{/ /}\right| / \mathrm{k}_{0}$ of the light emitted by the structure varies with the illumination conditions (unless specified otherwise, all the results presented in this study have been obtained at $\lambda=1350 \mathrm{~nm})$. The distributions recorded on Figs. 2(e)-(g) have been recorded by focusing a red $\mathrm{HeNe}$ laser pump at three different locations above the surface using an Olympus BX51XI microscope equipped with a 50X objective. The PL signal is collected with the same objective, separated from the pump with a dichroic mirror and a RG780 longpass filter, 
filtered with a 1350-nm bandpass filter (FWHM $12 \mathrm{~nm}$ ), Fourier-transformed with a 30-mm Bertrand lens and finally projected onto a Princeton NIRvana InGaAs camera using a 20-mm Telan lens. Because of the presence of the metal, the NCs excited by the laser spot do not emit light directly in free space. Rather, they act as a localized source of surface plasmons at the metal-air interface. As these modes propagate radially at the surface of the sample, they diffract in free space due to the corrugations. When the source of localized plasmons is excited at the center of the structure, a ring is formed, indicating that light propagates within a hollow cone [Fig. 2(e)]. As elaborated elsewhere ${ }^{19}$, this beam is a vector vortex beam with a topological charge equal to the number of branches of the spiral. Away from the center, the back focal plane images reveal the formation of arcs whose orientation depends on the position of the point excitation. This observation is consistent with the fact that, away from the singularity located at the center of the spiral, the corrugated Au surface resembles a deformed linear periodic grating.

We corroborate these observations with a scalar model originally developed to explain the formation of vector vortex beams in Ref. [19]. This model assumes that the PbS NCs excited by the focused laser spot act as a single point source from which a cylindrical wave is launched:

$A\left(x-x_{i}, y-y_{j}\right)=\exp \left[i n_{e f f} k_{0} \sqrt{\left(x-x_{i}\right)^{2}+\left(y-y_{j}\right)^{2}}\right] /\left[\left(x-x_{i}\right)^{2}+\left(y-y_{j}\right)^{2}\right]^{1 / 4}$,

where $\left(x_{i}, y_{j}\right)$ are the in-plane coordinates of the point source and $\mathrm{n}_{\mathrm{eff}}=1.012+0.003 \mathrm{i}$ is the complex effective index of the surface plasmon. This quantity is the only fitting parameter of our model. The diffraction of the surface wave with the grating is computed in the Fraunhofer approximation:

$$
I_{P L}\left(k_{x}, k_{y}\right)=\left|\mathcal{F}\left\{H(x, y) A\left(x-x_{i}, y-y_{j}\right)\right\}\right|^{2}
$$


where $H(x, y)$ is the function describing the grating geometry (see supplementary material) and $\mathcal{F}$ denotes the Fourier transform. $H(x, y)$ takes only two possible values: 0 for the bottom of the grooves and 1 for the top of the grooves. Figures 2(i)-(k) show that the different experimental cases are well captured by this model.

Figure 2(h) displays the emission pattern when the optical pumping almost covers the full area of the structure. This delocalized pumping is realized with the broadband halogen lamp and field illuminator of the microscope, using a band-pass filter to restrict the excitation wavelengths between 400 and $800 \mathrm{~nm}$ (i.e. far from the emission band of the PbS NCs). A field diaphragm within the illuminator is used to make the excitation area slightly smaller than the footprint of the spiraling structure. The emission pattern, plotted on Fig. 2(h), exhibits the same annular distribution as the vector vortex beam of Fig. 2(e), except that a much stronger isotropic background is now observed. This observation is expected and consistent with the results obtained with bull's eye structures in the context of thermal beaming ${ }^{16,17}$ and corrugated layers of $\mathrm{NCs}^{18}$, as discussed previously. By optically pumping the full structure, each $\mathrm{PbS}$ NC locally excites its own radial plasmons that are eventually diffracted in free space with a specific emission pattern [including those shown in Figs. 2(e)-(g)]. Because the NCs are not temporally synchronized, the ring pattern evidenced on Fig. 2(h) results from the incoherent superposition of all these lobes.

This situation can be reproduced with our model by evaluating the diffraction patterns produced by cylindrical waves regularly distributed over the whole area of the structure [including the diffraction patterns of Figs 2(i)-(k)] and by adding up the intensities of the lobes:

$$
I_{P L}\left(k_{x}, k_{y}\right)=\sum_{x_{i}, y_{j}}\left|\mathcal{F}\left\{H(x, y) A\left(x-x_{i}, y-y_{j}\right)\right\}\right|^{2} .
$$


Importantly, the resulting pattern, plotted on Fig. 2(1), is obtained even if one omits the contribution from the surface wave generated at the very center of the structure, which corresponds to the special case of the formation of a vector vortex beam. In other words, we do not observe a vector vortex beam, as on Figs. 2(e,i), but a composite ring produced by the superposition of many diffraction orders.

Although the predictions given by the model agree well with the experiments, there is a discrepancy regarding the background isotropic emission that accompanies the composite beam on Figs. 2(h,l). The background level observed in the experimental image is about two times higher, which we attribute to the imperfect coupling of the PbS NCs to the surface plasmons, resulting in direct unpolarized emission in free space. In contrast, the model assumes that all the energy is emitted under the form of radial waves. In this case, the sole origin of the background emission are the diffraction orders generated at all points of the structure, which concur to form the ring but also create a monotonous background everywhere else. This background, which has an intensity in every direction roughly equal to $20 \%$ of the maximum value reached at the emission angles of the composite ring, is part of the beaming mechanism and cannot be suppressed. It should be reminded, however, that the diameter of the ring depends on the wavelength since diffracting gratings are inherently dispersive. It is therefore possible to obtain a better contrast for wavelengths that lead to a narrower ring because the energy density is much higher in this case. Figure $2(\mathrm{~m})$ illustrates this point with the experimental and calculated dispersion relations of the structure under wide field illumination. The crossing branches evidenced in this diagram are nothing else than a cross-section of the composite beam as a function of the wavelength and one can see that their intensity can be much higher than the background around $\lambda=1250 \mathrm{~nm}$. 
We next repeat the wide field pumping experiments after inserting a linear polarizer in front of the InGaAs camera. Figure 3 shows that the beam is not a uniform ring anymore but that it features two lobes perpendicular to the polarization axis, regardless of the orientation of the latter. Thus, the composite beam originating from the extended light source has features reminiscent of a vector beam with radial polarization, the main difference being that the phase of the radial field varies randomly across the beam cross-section due to the incoherent contribution of all the points of the light-emitting surface. This observation reflects the fact that the sample can be seen as a combination of linear gratings with different orientations and that the diffraction of surface plasmons is TM polarized in the plane perpendicular to the ruling of such gratings ${ }^{25,26}$. Although easily explainable, such a complex polarization texture does not seem to have been reported by other authors who have studied incoherent sources. In particular, the formation of a composite beam with a well-defined radial electric field structure is different from the local variations of the linear polarization that have been observed above the corrugated surfaces studied in Ref. [15]. It is also noteworthy that the emission background discussed in the previous figure is also partially polarized because it has two contributions, one from the polarized diffraction orders and the other from the unpolarized light directly emitted by the NCs without being coupled to the surface plasmons.

Finally, we discuss the case of asymmetric spirals [Fig. 4(a)], characterized by a period that varies with the azimuthal angle. The height of the Au corrugations and the thickness of the PbS NC film coated onto it are the same as before. The structure has been designed to emit a tilted vector vortex beam at an angle $\alpha=10^{\circ}$ with respect to the normal when the focused laser spot excites the $\mathrm{PbS} \mathrm{NCs}$ covering the central geometric singularity. The experimental and calculated back focal planes images of Figs 4(b)-(c) validate the design approach when the structure is excited at its center, 
since the annular pattern of the vortex is not centered on the origin anymore but translated along the $\mathrm{ky}_{\mathrm{y}} / \mathrm{k}_{0}$ axis by a value of $\sin \left(10^{\circ}\right)$.

Figures 4(d)-(f) show the emission pattern obtained for a delocalized wide field pumping: both experiments and calculations reveal the formation of two rings, one at the same location as the vector vortex beam of Figs. 4(b)-(c), and the other centered on the opposite side of the ky axis. In addition, these rings are also radially polarized, as experimentally evidenced on Fig. 5. This symmetric pattern reflects the fact that the plasmons involved in the light emission are diffracted by a passive grating, implying that the distribution of the in-plane wavevectors (which again defines the emission angles) must be symmetric with respect to the origin.

This being said, we note that the relative strength of the two rings can be modulated by the size of the excitation area. The patterns of Figs. 4(d)-(f), for which the secondary ring is much weaker, haves been experimentally and numerically obtained by optically pumping $90 \%$ of the structure. On Figs. 4(g)-(i), in contrast, we have illuminated $225 \%$ of the structure, that is, we have also excited PbS NCs on the flat metal surface outside the spiral. In this case, the secondary ring is more intense than the primary one. This result indicates that the PbS NCs excited outside the structure play a role in the formation of the beam, by launching plasmons that are diffracted in free space when they reach the periphery of the grating. This interpretation is supported by our model that reproduces the same trends [compare Figs. 4(f) and 4(i)].

To conclude, we have shown that the directional beams created by large incoherent sources composed of PbS NCs coupled to plasmonic spirals have additional properties such as a radially distributed electric field. These conclusions do not depend on the type of emitters or surface waves or the method of pumping, implying that the same behavior would also be obtained for thermal 
emission and electroluminescence, thus enriching the nascent toolbox for the advanced control of incoherent light sources ${ }^{1-7}$. The main roadblock toward practical applications such as radially polarized LEDs is a sizeable isotropic background that may be mitigated by enhancing the coupling between the emitters and the surface plasmons.

\section{SUPPLEMENTARY MATERIAL}

See supplementary material for a description of the chemicals, the procedure followed to synthetize the $\mathrm{PbS}$ nanocrystals and the mathematical description of the grating function $H(x, y)$.

\section{ACKNOWLEDGMENTS}

We acknowledge support from the European Research Council Grant FORWARD (reference: $771688)$.

DATA AVAILABILITY STATEMENT

The data that supports the findings of this study are available within the article and its supplementary material. 


\section{REFERENCES}

${ }^{1}$ S. Inampudi, J. Cheng, M.M. Salary, and H. Mosallaei, J. Opt. Soc. Am. B 35, 39 (2018).

${ }^{2}$ P.P. Iyer, R.A. DeCrescent, Y. Mohtashami, G. Lheureux, N.A. Butakov, A. Alhassan, C. Weisbuch, S. Nakamura, S.P. DenBaars, and Jon.A. Schuller, Nat. Photonics 14, 543 (2020).

${ }^{3}$ Y. Mohtashami, R.A. DeCrescent, L.K. Heki, P.P. Iyer, N.A. Butakov, M.S. Wong, A. Alhassan, W.J. Mitchell, S. Nakamura, S.P. DenBaars, and Jon.A. Schuller, Nat. Commun. 12, 3591 (2021). ${ }^{4}$ A.Y. Song, A.R.K. Kalapala, R. Gibson, K.J. Reilly, T. Rotter, S. Addamane, H. Wang, C. Guo, G. Balakrishnan, R. Bedford, W. Zhou, and S. Fan, Appl. Phys. Lett. 119, 031105 (2021).

${ }^{5}$ M. Castro-Lopez, M. Gaio, S. Sellers, G. Gkantzounis, M. Florescu, and R. Sapienza, APL Photonics 2, 061302 (2017).

${ }^{6}$ K. Guo, M. Du, C.I. Osorio, and A.F. Koenderink, Laser Photonics Rev. 11, 1600235 (2017).

${ }^{7}$ A.C. Overvig, S.A. Mann, and A. Alù, Phys. Rev. X 11, 021050 (2021).

${ }^{8}$ R.E. Benner, R. Dornhaus, and R.K. Chang, Optics Communications 30, 145 (1979).

${ }^{9}$ W.L. Barnes, J. Lightwave Technol. 17, 2170 (1999).

${ }^{10}$ J.-J. Greffet, R. Carminati, K. Joulain, J.-P. Mulet, S. Mainguy, and Y. Chen, Nature 416, 61 (2002).

${ }^{11}$ J.M. Lupton, B.J. Matterson, I.D.W. Samuel, M.J. Jory, and W.L. Barnes, Appl. Phys. Lett. 77, 3340 (2000).

${ }^{12}$ M. Kreiter, J. Oster, R. Sambles, S. Herminghaus, S. Mittler-Neher, and W. Knoll, Opt. Commun. 168, 117 (1999).

${ }^{13}$ P. Andrew and W.L. Barnes, Phys. Rev. B 64, 125405 (2001).

${ }^{14}$ F. Marquier, C. Arnold, M. Laroche, J.J. Greffet, and Y. Chen, Opt. Express 16, 5305 (2008).

${ }^{15}$ N. Dahan, A. Niv, G. Biener, V. Kleiner, and E. Hasman, Appl. Phys. Lett. 86, 191102 (2005). 
${ }^{16}$ S.E. Han and D.J. Norris, Opt. Express 18, 4829 (2010).

${ }^{17}$ J.H. Park, S.E. Han, P. Nagpal, and D.J. Norris, ACS Photonics 3, 494 (2016).

${ }^{18}$ F. Prins, D.K. Kim, J. Cui, E. De Leo, L.L. Spiegel, K.M. McPeak, and D.J. Norris, Nano Lett. 17, 1319 (2017).

${ }^{19}$ D. Schanne, S. Suffit, P. Filloux, E. Lhuillier, and A. Degiron, Phys. Rev. Applied 14, 064077 (2020).

${ }^{20}$ I. Moreels, K. Lambert, D. Smeets, D. De Muynck, T. Nollet, J.C. Martins, F. Vanhaecke, A. Vantomme, C. Delerue, G. Allan, and Z. Hens, ACS Nano 3, 3023 (2009).

${ }^{21}$ I. Moreels, Y. Justo, B. De Geyter, K. Haustraete, J.C. Martins, and Z. Hens, ACS Nano 5, 2004 (2011).

${ }^{22}$ P. Bharadwaj, B. Deutsch, and L. Novotny, Adv. Opt. Photon. 1, 438 (2009).

${ }^{23}$ H. Wang, A. Aassime, X. Le Roux, N.J. Schilder, J.-J. Greffet, and A. Degiron, Phys. Rev. Applied 10, 034042 (2018).

${ }^{24}$ A. Caillas, S. Suffit, P. Filloux, E. Lhuillier, and A. Degiron, J. Phys. Chem. Lett. 12, 5123 (2021).

${ }^{25}$ M.C. Hutley and D. Maystre, Opt. Commun. 19, 431 (1976).

${ }^{26}$ H. Raether, Surface Plasmons on Smooth and Rough Surfaces and on Gratings (Springer Berlin Heidelberg, 1988). 


\section{FIGURE CAPTIONS}

FIG. 1. (a) SEM image of a three-branch spiral with a radial period of $1235 \mathrm{~nm}$ (all the other dimensions are given in the text). Scale bar: $1 \mu \mathrm{m}$. (b) PL spectrum of the uniform layer of PbS NCs covering the sample (there is an artifact around $1360 \mathrm{~nm}$ due to absorption lines of the atmosphere). Inset: schematic of the structure, with the PbS NCs represented as blue dots. (c)-(d) Schematic of the two pumping configurations. A HeNe laser is used to ensure a local excitation of the NCs, whereas the wide field illuminator of our microscope is used to excite the NCs on a more extended area. The coupling of the PbS NCs to the plasmons is illustrated by the radial arrows with an "evanescent" tail and the blue arrows represent the PL signal diffracted by the spiral.

FIG. 2. (a)-(d) Sketches visualizing the different pumping conditions. The NCs are either excited by (a)-(c) a laser beam focused at different positions above the spiral or (d) by a wide field lamp illuminating the entire structure. The actual diameter of the spiral $(100 \mu \mathrm{m})$ is much larger than in these drawings. (e)-(h) Experimental images of the back focal plane obtained for each pumping conditions. (i)-(l) Corresponding theoretical images. All data have been obtained at $\lambda=1350 \mathrm{~nm}$. The white circle of these plots has a radius $\mathrm{k} / \mathrm{k}_{0}=0.65$ corresponding to the numerical aperture of the objective. $(\mathrm{m})$ Dispersion relation obtained experimentally (negative values) and theoretically (positive values) under a wide field illumination of the structure. The color bar applies to all plots.

FIG. 3. Back focal plane polarization measurements obtained for a wide field illumination of the symmetric spiral. The double arrows plotted on each panel indicates the orientation of the linear polarizer.

FIG. 4. (a) SEM close-up of the asymmetric spiral, with a scale bar of $1 \mu \mathrm{m}$ and a red dot marking the location of the focused laser beam for the theoretical and experimental back focal plane images of panels (b)-(c). (d)-(f) Experimental and theoretical back focal plane images obtained by pumping $90 \%$ of the 
structure and (g)-(i) $225 \%$ of the structure. The actual diameter of the spiral $(100 \mu \mathrm{m})$ is much larger than the drawings.

FIG. 5. Back focal plane polarization measurements obtained for a wide field illumination of the asymmetric spiral. The diaphragm in front of the pumping white lamp is fully open to excite all the QDs on the spiral but also in its vicinity. This enables a better visualization of the polarization states of the secondary ring, as demonstrated on Fig. 4. 

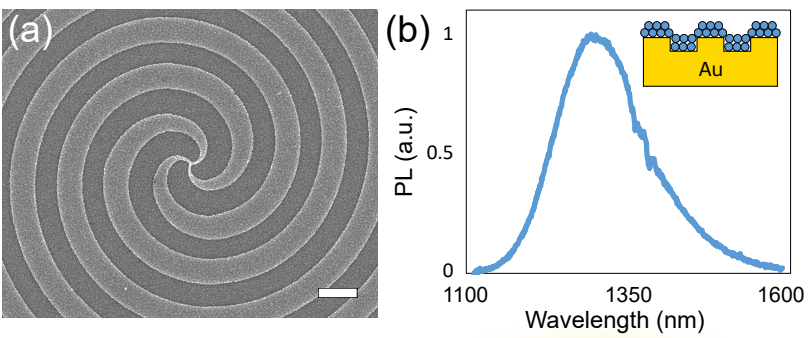

(c)

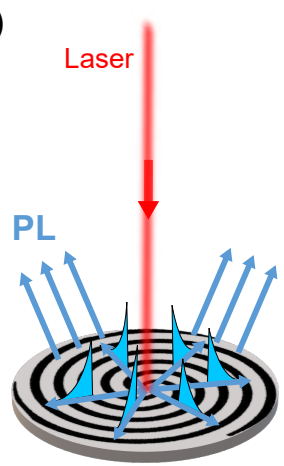

(d)

Wide field pumping

PL<smiles>C1CCCC1</smiles>

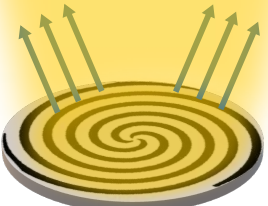




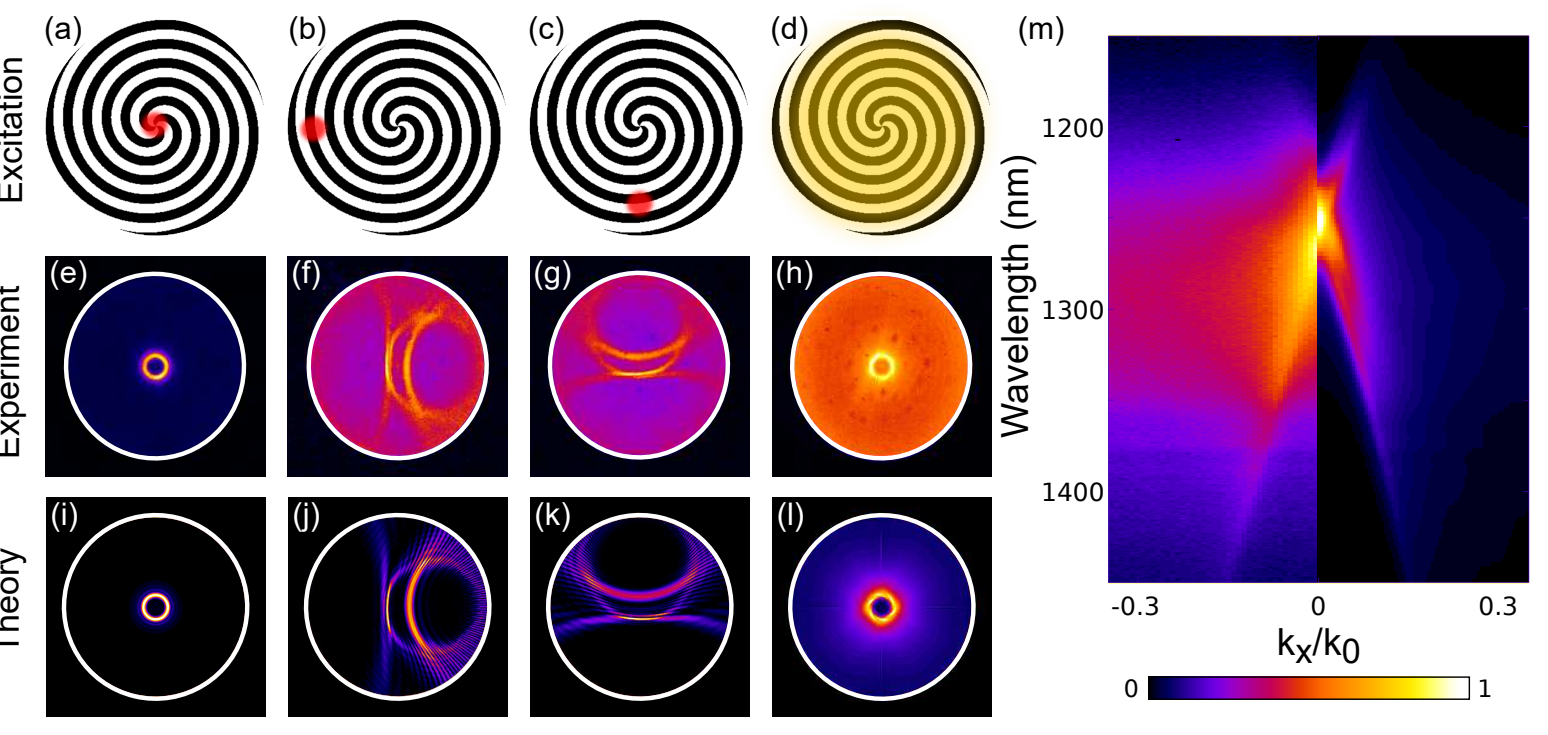



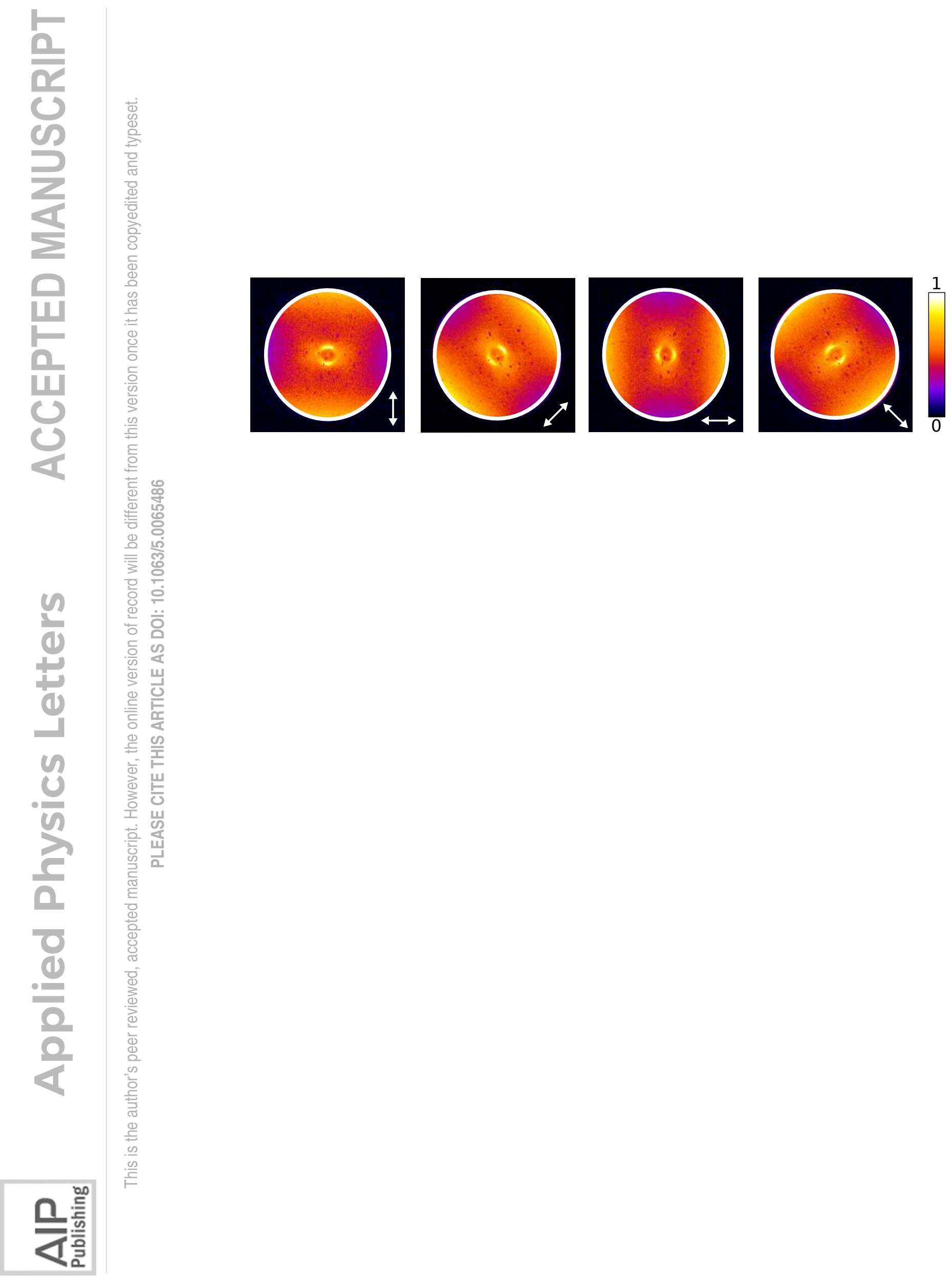

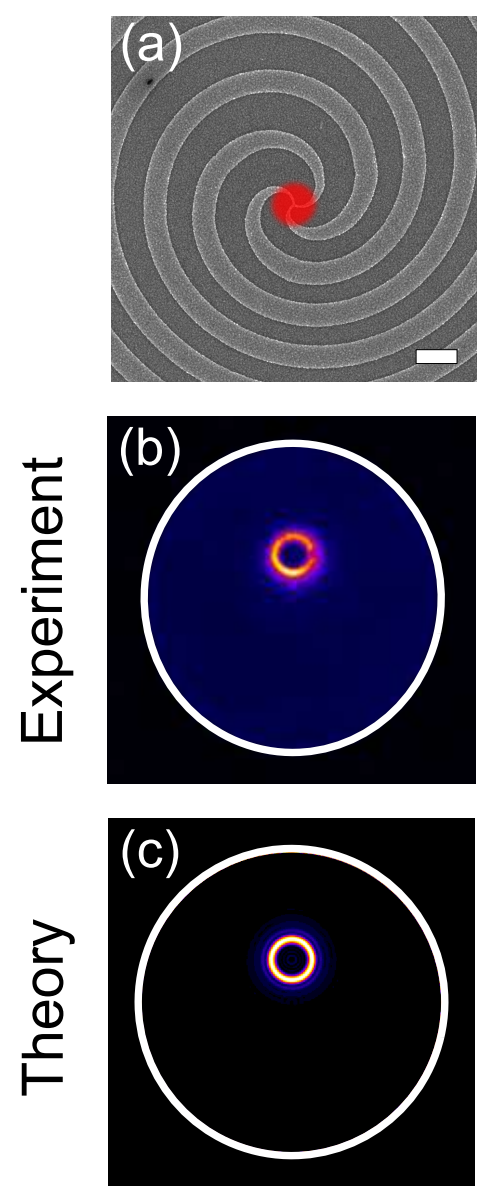

(d)

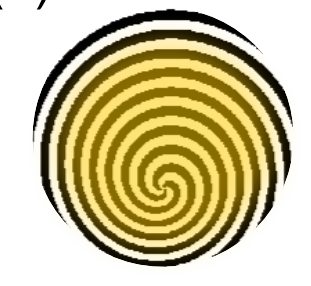

(g)
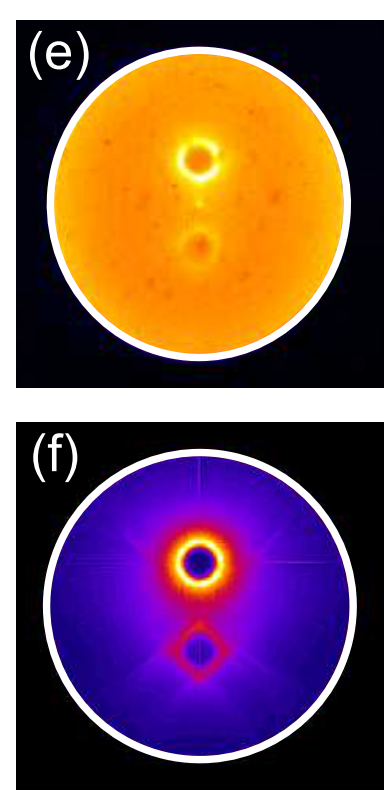
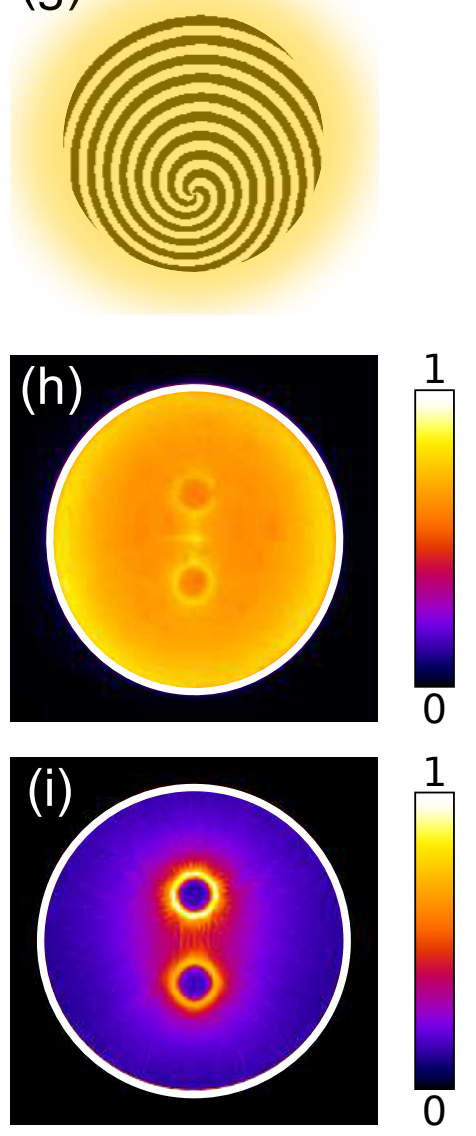

兄喜产 

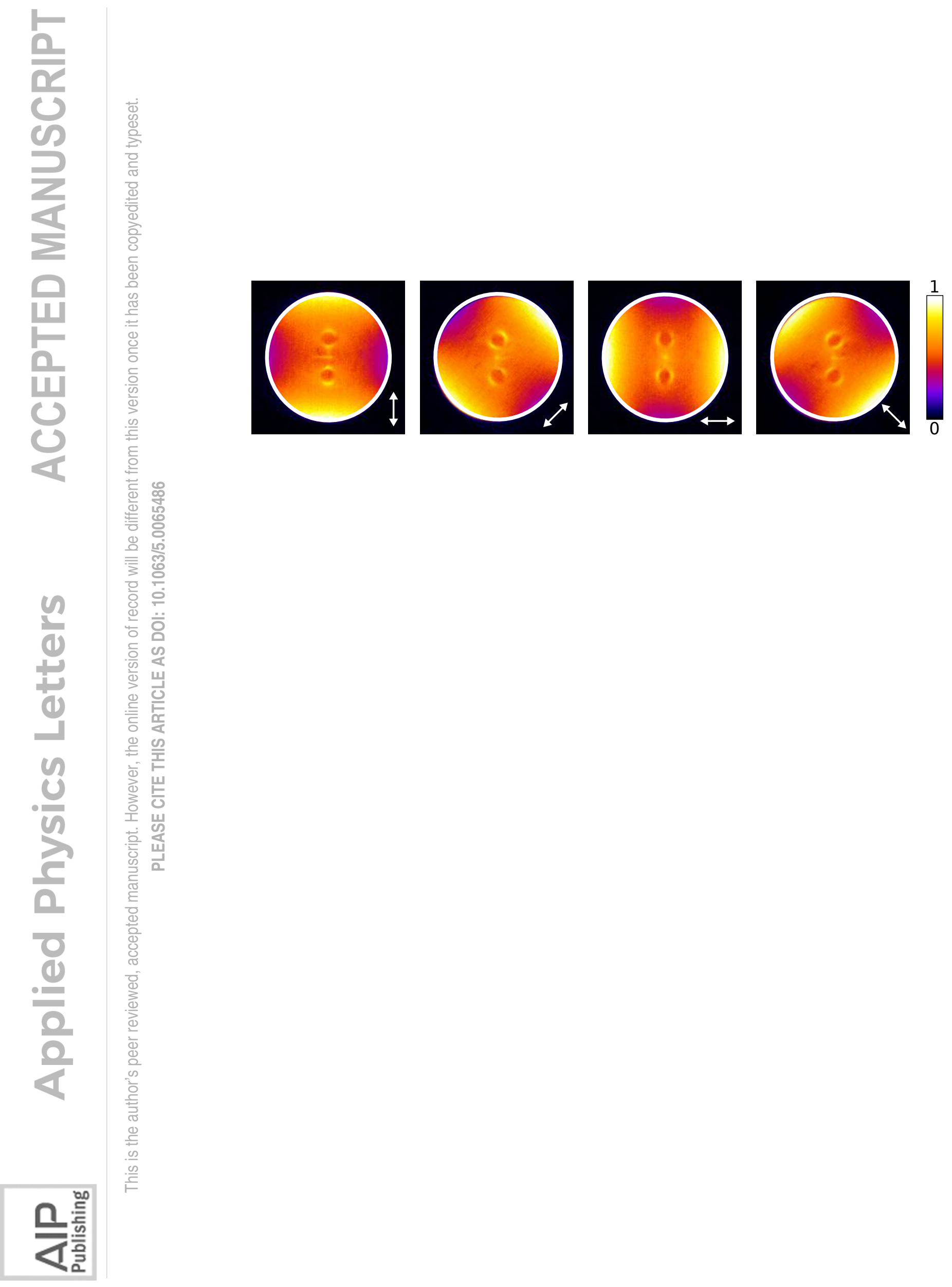